\title{
FDM 3D Printed Coffee Glove Embedded with Flexible Electronic
}

\author{
Meznan Bahri \\ Undergraduate Student \\ ECE Department \\ Effat University \\ Jeddah, Saudi Arabia \\ mgbahri@effat.edu.sa
}

\author{
Muhammad M. Hussain \\ Associate Professor \\ EE Department \\ KAUST \\ Thuwal, Saudi Arabia \\ MuhammadMustafa.Hussain@ \\ kaust.edu.sa
}

\author{
Tayeb Brahimi \\ Assistant Professor \\ ECE Department \\ Effat University \\ Jeddah, Saudi Arabia \\ tbrahimi@effatuniversity.edu.sa
}

\author{
Hayssam Dahrouj \\ Assistant Professor \\ ECE Department \\ Effat University \\ Jeddah, Saudi Arabia \\ hdahrouj@effatuniversity.edu.s
}

\begin{abstract}
With the advances in 3D printing technology, Flexible Electronics can now be exploited to form the so-called "Embedded Electronics". This paper describes experiences learned from a research project which ran during summer 2016 at KAUST, in collaboration with the Electrical and Computer Engineering Department at Effat University, and aimed at creating a heating coffee glove product operating on double alkaline batteries using Kapton(C) as a flexible substrate for the circuit. The circuit and its batteries are encapsulated in a 3D printed glove, designed using SolidWorks $\odot$. The proposed methodology and techniques applied during this work could be further used in implementing other technologies, such as thermoelectric coolers head patches, smart garments, and flexible smartphones. Limitation and recommendation of the present methodology are also discussed.
\end{abstract}

Keywords-Flexible Electronics, Embedded Electronics, 3D Printing, Fused Deposition Modeling, SolidWorks $\odot$.

\section{INTRODUCTION}

Unlike conventional semiconductor electronics that are limited to rigid wafers, a timely topic of study is Flexible Electronics-as it provides the required malleability and durability $[1,2]$. In recent years, many programs have been launched in US, Asia, and Europe [3] to develop the new technology of Flexible Electronics; ranging from energy sustainability to smart sensor, and to complex curvilinear stretchable devices for biomedical applications as reported by Ko et al. [4]. With the help of 3D printing [5, 6], Flexible Electronics can be further exploited to form the so-called "Embedded Electronics". From one side, the technology of Flexible Electronics benefits from its low production cost, low energy consumption, environmentally friendly materials, and wearable and bendable designs. From the other side, 3D printing follows an excellent process capable of creating geometrically complex designs not previously possible with traditional techniques. It is also improved in quality, decreased in cycle time, reduced in costs, as well as customized and personalized in products with a high energy efficiency and low-waste creation factors; thereby eliminating the need for tools production. The present study amalgamates the advantages of Flexible Electronics and $3 \mathrm{D}$ printing so as to design a product that can keep the hot beverage warm for a longer time-period. This project, performed in the Nanotechnology Lab facilities at KAUST, in collaboration with the Electrical and Computer Engineering Department at Effat University, aimed to deliver a heating coffee glove product operating on double Alkaline AA $1.5 \mathrm{~V}$ batteries. Its outcome is to maintain the heat of the hot beverage for a longer period while protecting human hands from the heat of the circuit. The present paper is intended to describe the project by thoroughly describing the experiments goals, setup, and results as well as presenting the project conclusion and shedding light on future research directions.

\section{Fused Deposition Modeling (FDM)}

Scott Crump [7], co-founder and chair of Stratasys, a leading manufacturer of $3 \mathrm{D}$ printers, invented $3 \mathrm{D}$ printing also known as additive manufacturing (AM) in the 1980s. Other 3D printing organizations used similar technologies, known as Fused Filament Fabrication (FFF), such as MakerBot which is now owned by Stratasys Inc. Since then, this technology is becoming more and more popular succeeding the production line assembly of the $19^{\text {th }}$ century. According to the Wohlers Report $2016[6,8]$, the 3D printing industry grew by $25.9 \%$ year over year in 2015 , generating nearly $\$ 5.2$ billion in worldwide revenue. One of the 3D printing techniques developed and manufactured by Stratasys, Inc. is called Fused Deposition Modeling (FDM) or Fused Filament Fabrication (FFF) $[9,10,11]$. This method is particularly characterized by a heated nozzle that extrudes the chosen material filament in 3D space, layer by layer. Using this method, the 3D printer takes the thermoplastic material filament; extrudes it through an extrusion head that deposits the molten plastic in $\mathrm{X}$ and $\mathrm{Y}$ directions while the object parts are built layer by layer in the $\mathrm{Z}$ direction, from the bottom up. FDM is the most common 3D printing method and has become increasingly used as a tool for producing manufactured products. Other 3D printing technologies can be found in the literature [11] such as: i) Stereolithography (SLA) where a layer of photosensitive liquid resin is exposed to a UV-laser beam and the resin becomes solid; ii) The Selective Laser Sintering (SLS) which uses powdered material instead of liquid resin; and iii) The Electron Beam Melting (EBM) which uses a computer-controlled 
electron beam to melt the metallic powder at high temperatures up to $1000{ }^{\circ} \mathrm{C}$.

For any 3D printing technology, a computer-aided design (CAD) program [12] is required to build and create the model design, such as: AutoDesk, AutoCAD, SolidWorks, or Creo Parametric. In this project, SolidWorks software was used to design the object of the coffee glove, converted into a ".STL" file, in order to be read by the slicing program. The slicing program used was Simplify3D, which would send binary data to the $3 \mathrm{D}$ printing machine.

\section{CIRCUIT DESIGN}

Flexible Electronic devices are fabricated with the help of nanotechnology. For this particular project, nanoparticles of silver were used to design a circuit on the flexible substrate. Firstly, the substrate needs to be cleaned using suitable cleaning solvents (such as IPA). Then, pre-treatment is performed on a Kapton $\odot$ sheet to deliver better adhesion between the silver and the substrate. Dry etching can be used in such case, and it is a material removal process, which ionizes gasses in order to attract them to the charged plate beneath the substrate, hence etching its surface. The gasses used are $\mathrm{CF}_{4}$ and $\mathrm{O}_{2}$ with ratio 25/5. UVO, Fig. 1 , is a damage-free alternative to removing organic contaminants. UVO cleaning pre-treatment can be used instead of dry etching, Fig. 2, to deliver better adhesion, which a photosensitized oxidation process in which the contaminant molecules of photo resists, resins, human skin oils, cleaning solvent residues, silicone oils, and flux are excited and/or dissociated by the absorption of short-wavelength UV radiation.

After having the substrate treated, silver ink is deposited using an inkjet printer, then cured in the oven at a temperature of around $130^{\circ} \mathrm{C}$ for the Jetlab silver ink, and $250^{\circ} \mathrm{C}$ for the Dimatex ink. The pattern of the circuit is shown in Fig. 3.

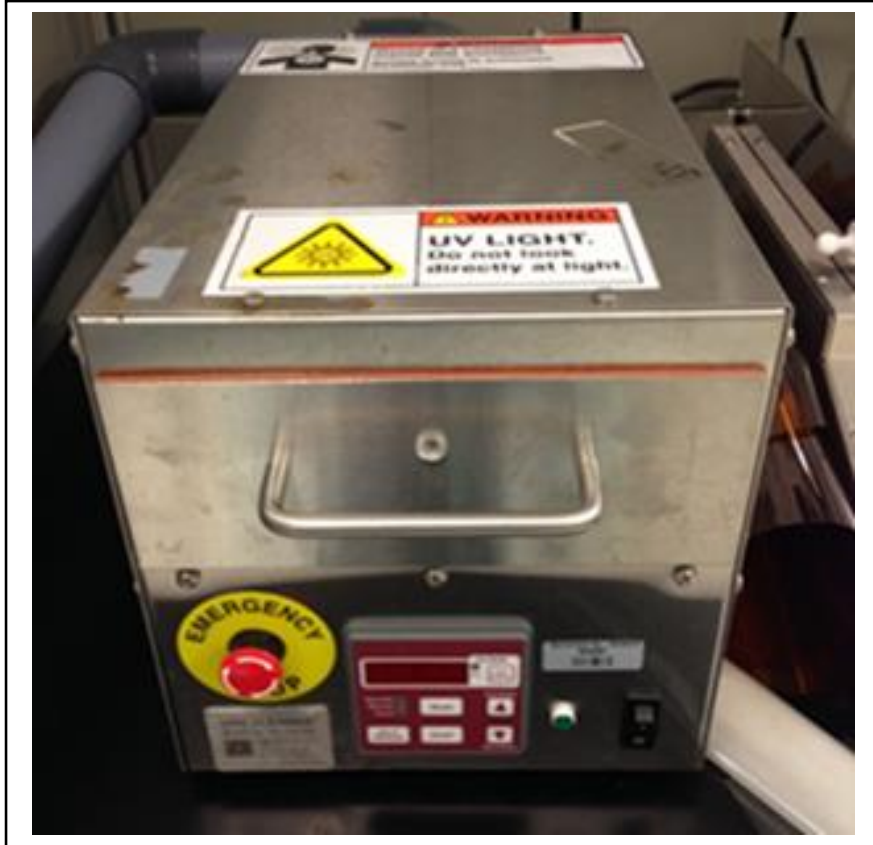

Fig. 1. UVO Cleaner

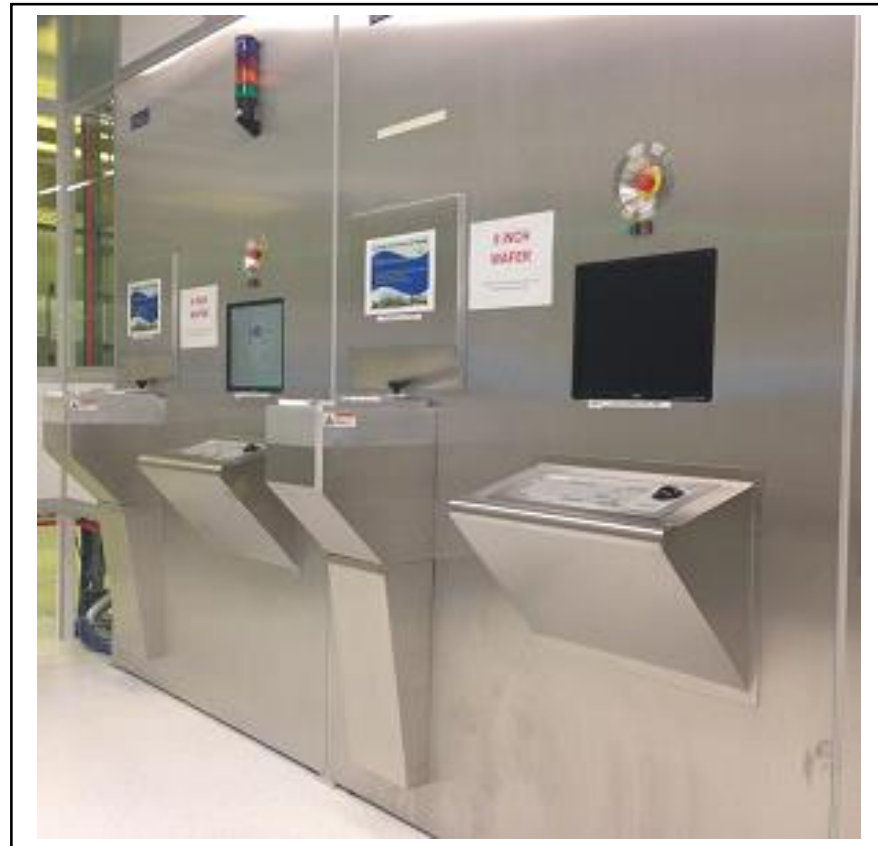

Fig. 3. Dry Etching Machines

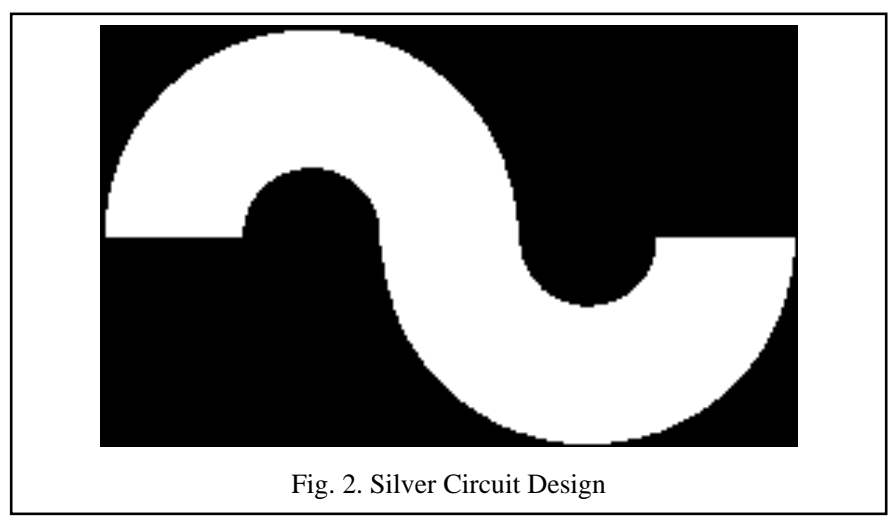

The ends of the circuit design are connected to two wires, for the battery connections. Afterward, the circuit's resistance is measured to calculate its resistivity using the following equation:

$$
R=\frac{\rho L}{A}
$$

Where $\rho_{x} L_{x}$ and $A$ are resistance, resistivity coefficient, length, and area, respectively. The circuit is connected to the specified batteries and compares it to the theoretical heat value. Using Kapton $\odot$ as a flexible substrate for the circuit, silver is deposited on it and acts as a conductor to serve the purpose of Ohmic, Resistive, or Joule heating. The form of silver deposition is in a liquid state using Inkjet Printing, processed by spraying very fine drops on top of a board or substrate. 


\section{PROTOTYPE DESIGN}

After testing the heat dissipation from the circuit samples, the 3D design is then printed, Fig. 4. It has designated battery slots in the bottom part, with a sliding lid to cover the batteries, and circuit substrate designated slot as well, which is embedded in the walls of the coffee glove model by pausing, inserting the circuit between the inner pillars of the coffee glove and placing it in its slot, then resuming the 3D printing process. The printed design is shown in Figs. 4 and 5.

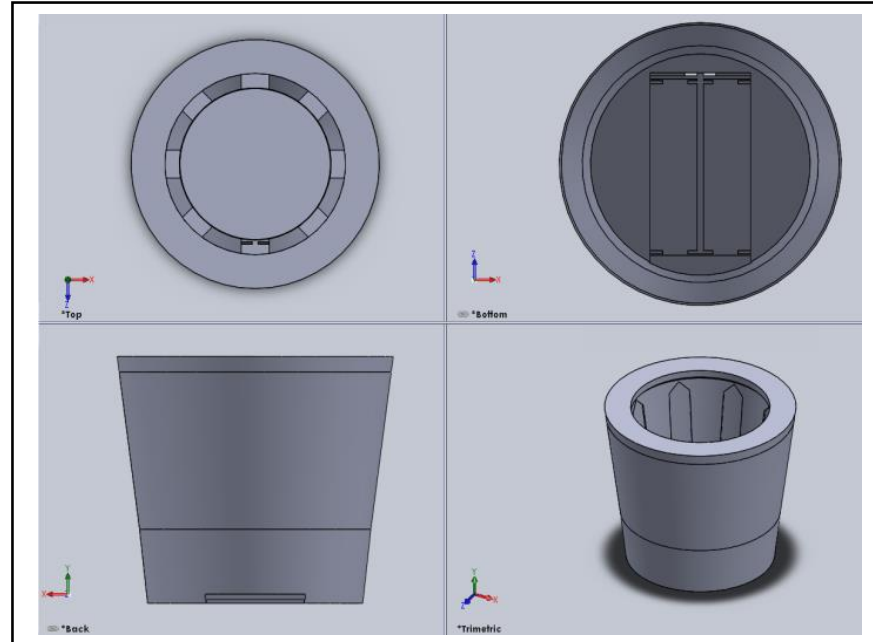

Fig. 6. Different Views of the 3D Glove Design

\section{Data Analysis}

Data discussed in this section govern the silver ink named DGH 55LT-25C [13], which has a solid content of silver of $50 \sim 60 \%$ and a curing temperature of $250^{\circ} \mathrm{C}$, used with Jetlab inkjet printer shown in Fig. 6., in addition to the silver ink named DGP 40LT-15C [13], with 30 35\% solid content of silver and a curing temperate of $120^{\circ} \mathrm{C} \sim 150^{\circ} \mathrm{C}$, used with the Dimatex inkjet printer shown in Fig. 7. It can be observed from Figs. 8 and 9 that the heat has increased with the Jetlab ink due to the increase in thickness. Also, it is less time consuming since multiple layers are discarded. However, a much higher temperature is needed to cure the new ink. The limit of the power source (batteries) also limits the ability to use the circuit as one piece.

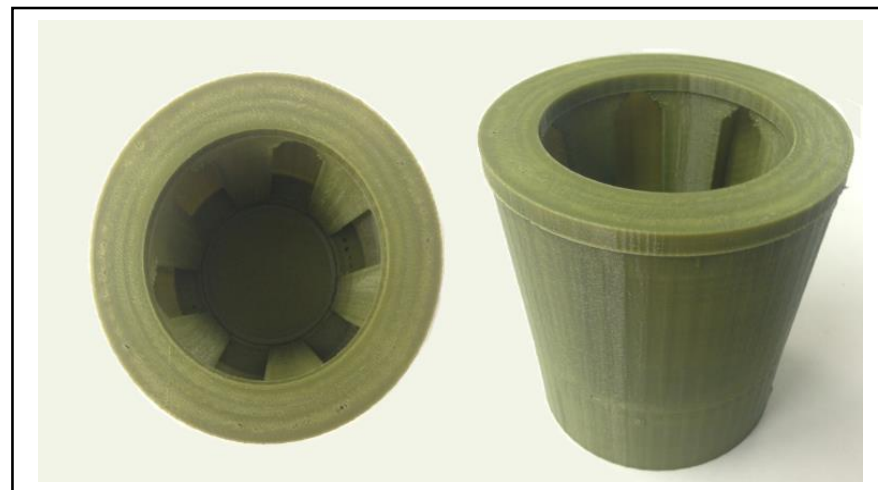

Fig. 8. Printed Version of the 3D Glove Design
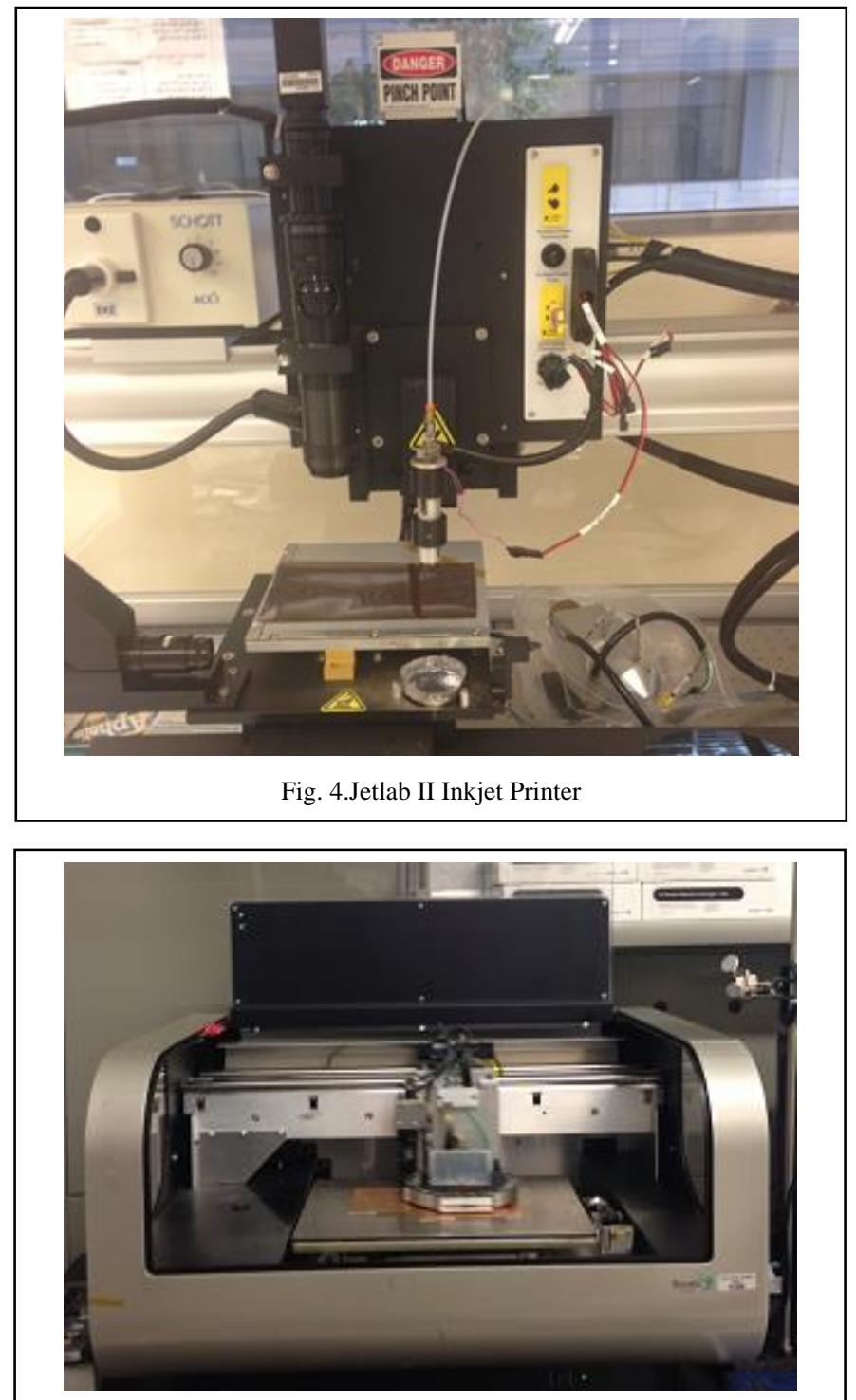

Fig. 5. Dimatex Inkjet Printer

\section{Dimatex Circuit Sample}

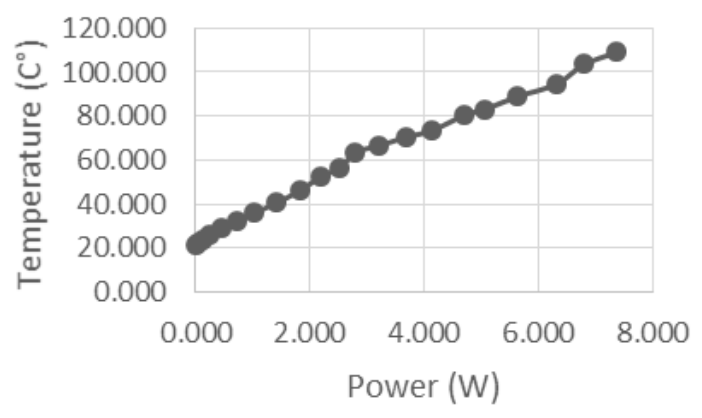

Fig. 7. Dimatex Circuit Sample Heat VS Power 


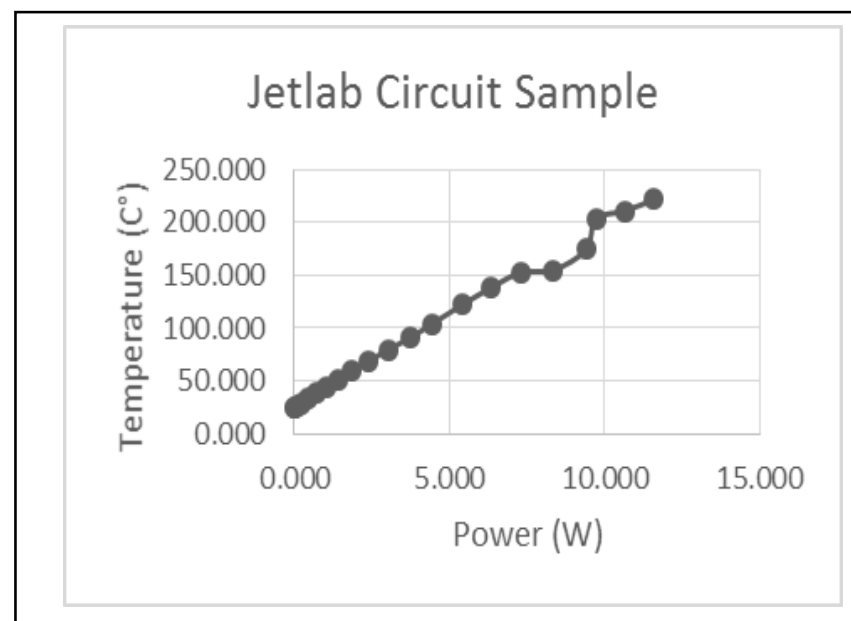

Fig. 9. Jetlab Circuit Sample Heat VS Power

Although the new ink was relatively better in heat, each ink and their corresponding inkjet printers have advantages and disadvantages. For Jetlab II inkjet printer sample in in Fig. 10 , its lack of accuracy led to many problems, which was hard to follow up with since it needed to have its settings modified frequently with different parameters, and the need for cleaning its nozzle with every use. The reason goes back to the tool being old, the nozzle being wide (60 microns) and the technique used (pressure pumping). However, it saved more time by needing only one layer. As for Dimatex and its lowsolid content silver ink in Fig. 11, the sample required multiple layers of which each needed curing, resulting in long periods of time to create each sample. But they were very well adhered and evenly distributed due to the accuracy of the machine with a small nozzle (30 microns) using piezo-electric technique.

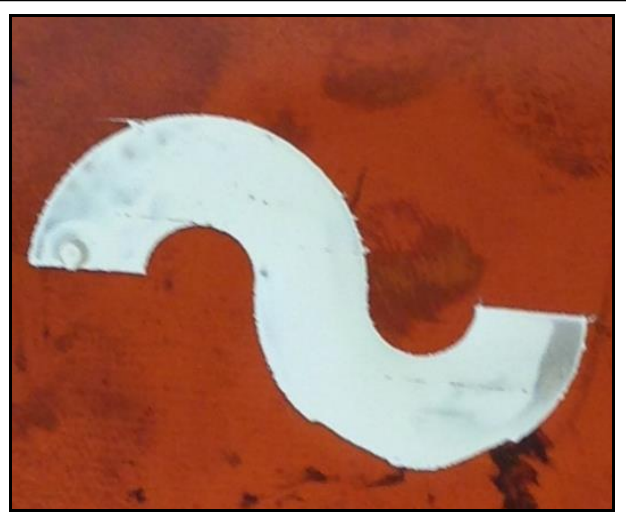

Fig. 10. Jetlab Circuit Sample Heat VS Power

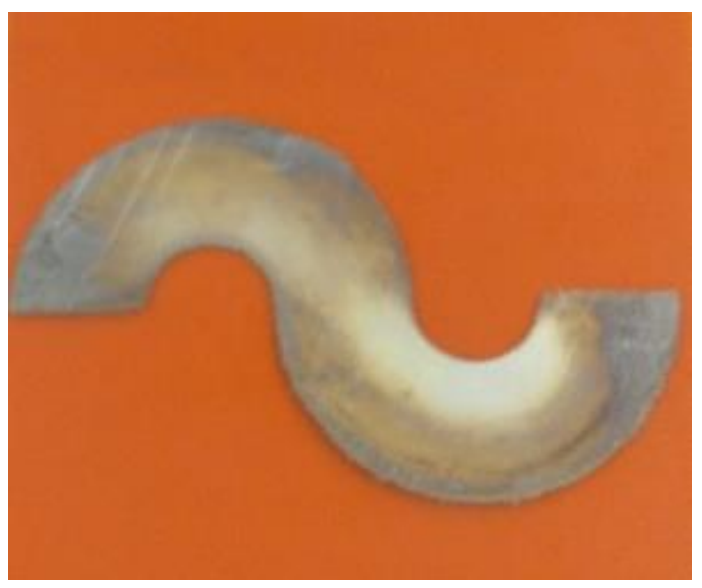

Fig. 11. Dimatex Circuit Sample Heat VS Power

\section{CONCLUSION AND RECOMMENDATION}

The present paper described experiences learned from a research project conducted at KAUST, in collaboration with the Electrical and Computer Engineering Department at Effat University, and aimed at creating a heating coffee glove product that can keep the hot beverage warm for a longer time-period. Experiments, goals, setup and results have been presented. The proposed methodology and techniques applied during this work can be further used in implementing other technologies, such as thermoelectric coolers head patches, smart garments, and flexible smartphones. Both the 3D printer and the Inkjet printers required tuning the machines, monitoring the process, and understanding how to improve the printed design. The accuracy of the $3 \mathrm{D}$ printing machine was acceptable for experimental causes, but not for market use. As for the inkjet printers, Dimatex was more accurate than Jetlab II, but more time consuming, due to the inkjet printer's accuracy and time issues.

\section{ACKNOWLEDGMENT}

The present project was prepared under the collaboration of KAUST and the Electrical and Computer Engineering Department at Effat University. The authors gratefully acknowledge their support and express their deepest thanks to KAUST for arranging all facilities to complete this project.

\section{REFERENCES}

[1] M. Caironi, Y.-Y. Noh, "Large Area and Flexible Electronics". WileyVCH Verlag GmbH \& Co. KGaA, 2015

[2] Logothetidis, S., "Handbook of Flexible Organic Electronics", Materials, Manufacturing and Applications, Woodhead Publishing Series in Electronic and Optical Materials: Number 68, 2015

[3] National Research Council, "The Flexible Electronics Opportunity", Committee on Best Practice in National Innovation Programs from Flexible Electronics, National Academies Press, 2015

[4] Ko, H. C., Shin, G., Wang, S. D., Stoykovich, M. P., Lee, J. W., Kim, D. H., "Curvilinear electronics formed using silicon membrane circuits and elastomeric transfer elements", Small, 2009, Vol 5. No 23, pp . 27032709 
[5] Ota H., Emaminejad S., Gao Y., Zhao A., Wu E., Challa S., Chen K., Fahad H. M., Jha A. K., Kiriya D., Gao W., Shiraki H., Morioka K., Ferguson A. R., Healy K. E., Davis R. W., Javey, "Application of 3D Printing for Smart Objects with Embedded Electronic Sensors and Systems". Advanced Materials Technologies Volume 1, Issue 1, 2016, DOI: $10.1002 / \mathrm{admt} .201600013$

[6] Heller, S., "Is Competition Hurting 3D Systems and Stratasys?". Retrieved November 2016, from http://www.fool.com/investing/2016/05/27/is-competition-hurting-3dsystems-and-stratasys.aspx

[7] Crump, S., Inventor of 3D Printing Scott Crump: "My Dreams Started in a Garage", Inside 3D Printing Conference Kicks Off in San Jose, 2013. Retrieved November 2016 from http://sumo.ly/fJb

[8] Wohlers Report 2016, "A Worldwide Review and Analysis of Additive Manufacturing and 3D printing. Retrieved November 2016 from https://wohlersassociates.com/2016report.htm

[9] Hiemenz, J.. "3D Printing With FDM, A Guide to the FDM process and its application". Retrieved November 2016, By Joe Hiemenz, Stratasys, Inc.http://www.stratasys.com/3d-printers/technologies/fdm-technology.

[10] Grimm, T., "Fused Deposition Modeling: A Technology Evaluation", Grimm \& Associates, Inc. Retrieved November 2016, from http://tagrimm.com/downloads/fdm-white-paper.pdf

[11] All3DP, "7 Essential Types of 3D Printers Explained" (A Guide)". Retrieved November 2016, from https://all3dp.com/types-of-3d-printertechnology-explained/

[12] Gross, B. C., Erkal, J. L., Lockwood, S. Y., Chen, C., \& Spence, D. M. (2014). Evaluation of 3D printing and its potential impact on biotechnology and the chemical sciences., Anal. Chem., 2014, 86 (7), pp 3240-3253, DOI: $10.1021 / \mathrm{ac} 403397 \mathrm{r}$

[13] ANP (2016). Nano-Silver Ink for Inkjet Printing. Retrieved from http://anapro.com/eng/product/silver_inkjet_ink.html 